\title{
Reliability of a freehand three-dimensional ultrasonic device allowing anatomical orientation "at a glance": Study protocol for 3D measurements with Curefab CS ${ }^{\circledR}$
}

\author{
Regina Feurer ${ }^{1}$, C. Hennersperger ${ }^{2}$, J .B. Runyan ${ }^{3}$, C.L. Seifert ${ }^{1}$, J. Pongratz ${ }^{4}$, M. Wilhelm ${ }^{4}$, J . \\ Pelisek $^{4}$, N. Navab ${ }^{2}$, E. Bartels ${ }^{5}$, H. Poppert ${ }^{1}$ \\ 1. Department of Neurology, Technische Universitaet Muenchen, Klinikum rechts der Isar, Muenchen, Germany. 2. Chair \\ for Computer Aided Medical Procedures (CAMP), Technische Universitaet Muenchen, Germany. 3. Curefab GmbH, \\ Muenchen, Germany. 4. Department of Vascular Surgery, Technische Universitaet Muenchen, Klinikum rechts der Isar, \\ Muenchen, Germany. 5. Department of Clinical Neurophysiology, Georg-August Universitaet Goettingen, Germany. \\ Correspondence: Regina Feurer. Address: Department of Neurology, Klinikum rechts der Isar, Technische Universitaet \\ Muenchen, Ismaningerstr. 22, 81675 Muenchen, Germany. Telephone: 0049-894-140-4645. Email: \\ regina.feurer@gmx.de.
}

Received: January 23, 2012

DOI : $10.5430 /$ jbgc.v2n2p1
Accepted: May 17, 2012

Published: December 1, 2012

URL: http://dx.doi.org/10.5430/jbgc.v2n2p1

\section{Abstract}

Background and Purpose: 3D ultrasonic measurement of carotid atherosclerotic lesions has emerged as an important tool for research and patient management. We sought to evaluate the accuracy and the reliability of a new easy-to-use magnetically tracked freehand 3D ultrasonic device with quick reconstruction time, allowing for anatomical orientation "at a glance" for calibration and distance measurements, with unique usability as a combined tool for image acquisition, registration and measurement within 3-D ultrasonic (US) volumes, which is furthermore connectable to every conventional US-machine. To enable a complete evaluation of the whole system, three different key points had to be addressed: the reproducibility of the calibration procedure, the absolute accuracy of the whole system and a direct comparison to CT and MRI imaging modalities.

Materials and Methods: For validating our calibration method, a set of 6 calibrations was performed; each consisting of 6 records of a pyramid phantom taken from different positions and angles. To evaluate the accuracy of the whole 3D-ultrasound system, the point reconstruction accuracy and the distance accuracy were determined in a point phantom made out of a single metal wire vertically attached to the bottom of a plastic tub filled with water. For distance measurements, a precisely manufactured plastic tube phantom was scanned and the length between fixed landmarks on the tube was measured. In a final step, 3D US records acquired with Curefab CS were compared to CT and MRI scans; for this purpose all ultrasonic data was manually registered to the CT/MRI data.

Results: Concerning calibration precision the tested Curefab CS system performs state of the art compared to reviews of recent freehand 3D-Ultrasound calibration methods. The point reconstruction measure for evaluation of system accuracy retrieved a mean point accuracy of $1.52 \mathrm{~mm}$ in contrast to values ranging from 1.67 to $3.63 \mathrm{~mm}$. Mean total error of distance measurements was $0.9 \%$ with standard deviation $0.56 \%$ in our study, compared with values reaching from about $1 \%$ up to $2.3 \%$ in other studies on this subject. All quantitative measurement results are listed in a summarized form in 
Table 1. Besides quantitative evaluation, 3D-ultrasound records acquired with the Curefab CS system were also compared to CT and MRI scans of patients (see Figure 3 and Figure 4). The alignment of both image modalities showed promising results for future development of diagnostic tools using all image data.

Discussion and Conclusion: Our study demonstrates that 3D measurements with Curefab CS are feasible with satisfactory reliability and accuracy. From the results gathered in our study we conclude that 3D-imaging with Curefab CS might start off the possibility of accurate visualization, volume measurement, carotid plaque characterization and identification of vulnerable plaques in the very near future.

\section{Key words}

3-D ultrasound, Ultrasound probe calibration, Distance measurements, Freehand acquisition

\section{Introduction and background}

Whereas up to now the detection of the severity of carotid atherosclerotic stenosis has been considered crucial for establishing patient management pathways and selecting patients who might benefit from carotid endarterectomy instead of drug intervention and lifestyle adaption only, other phenotypes than carotid artery stenosis developed by usage of noninvasive imaging have evolved recently for recognizing patients at high risk for ischemic stroke ${ }^{[1]}$. Monitoring the progression or regression of carotid plaques with three-dimensional ultrasound imaging by identifying vulnerable and high-risk plaques prone to rupture has emerged as an important research tool for antiatherosclerotic treatment and patient management ${ }^{[2]}$. Conventional ultrasound measurements, such as the 1-D measurement of the intima-media thickness and the 2-D measurement of plaque surface, have proven useful for monitoring carotid atherosclerotic burden ${ }^{[3]}$. Conventional 2-D US has also been applied for relating plaque morphology and component parts with the risk of stroke ${ }^{[1]}$, although the results have not always been consistent. While some studies have reported a high precision for visualizing components such as intraplaque hemorrhage ${ }^{[4,5]}$, others have found only moderate sensitivity and specificity ${ }^{[6]}$.

Hence, while there seems to be general agreement that plaque morphology is equally as important as the degree of stenosis in assessing stroke patients at risk, better US techniques will have to be developed for visualization and measurement. Three-dimensional ultrasound seems to be the desired development which might provide the capability of improving the visualization and quantification of complex anatomic structures by overcoming the following limitations of conventional ultrasound, as described by Fenster et al. ${ }^{[3,7]}$ :

a) As conventional US images are two-dimensional, a mental impression of the complex three-dimensional anatomy has to be formed out of multiple 2-D images by the examiner, leading to variable or incorrect assessment.

b) Conventional 2-D US images are difficult to reproduce and are therefore nonoptimal for prospective or follow-up studies, particularly when referring to small changes over the course of time.

c) Location and orientation of a conventional ultrasound image is sometimes limited by patient's anatomical conditions, resulting in inapproachability of the requested image plane.

Routine application of three-dimensional ultrasound is confined to usage in obstetrics (e.g., imaging of the fetus), cardiology (e.g., imaging of the cardiac system) and image guidance (e.g., imaging of the prostate during an interventional procedure $)^{[8,9]}$. Despite its usefulness for noninvasive plaque imaging of the carotid arteries, risk stratification and genetic research $^{[10,11]}$, evaluation of response to antiatherosclerotic and antihypertensive therapy ${ }^{[12,14]}$ and evaluation of the influence of suspected risk factors ${ }^{[15,16]}$, the modality of three-dimensional ultrasound has not yet been established as a routine diagnostic tool on ultrasound machines. One possible explanation for this may be the fact that images of the carotid arteries comprise a scanning length of at least $4 \mathrm{~cm}$, so that real-time 3-D systems cannot be used satisfactorily ${ }^{[1]}$. Besides these real-time 3-D probes, various other 3-D ultrasound systems have been presented recently. The different approaches 
can be subdivided into (i) mechanical scanning devices where a part of the ultrasound probe or the whole itself is moved ${ }^{[9]}$, (ii) freehand 3D ultrasound devices without the use of a tracking system, which make use of specific image parameters such as speckle or echo intensities to register the series of $2 \mathrm{D}$ frames ${ }^{[17,18]}$ and (iii) freehand $3 \mathrm{D}$ ultrasound systems with the use of a tracking system, which represents the most frequently used technique ${ }^{[19-23]}$. For pose (i.e., position and orientation) information, any tracking system which provides the position and orientation of a sensor in 3D space can be used. In practice, electromagnetic and optical tracking systems are used most frequently. For a detailed overview of all 3D ultrasound approaches, the reader is referred to ${ }^{[8,9,22,23]}$. A major drawback of almost all these recently proposed systems is that current visualization tools often demand complicated user interfaces and special training for the diagnostician, which is not feasible in clinical routine.

We therefore focus on evaluating the reliability of a new easy-to-use freehand three-dimensional ultrasonic device with quick reconstruction time, allowing for rapid anatomical orientation, for prospective non-invasive monitoring of atherosclerotic disease. To the best of our knowledge the device presented here offers the unique usability as a combined tool for image acquisition and registration, as well as measurement as the following step within 3-D US volumes. Curefab CS is a three-dimensional ultrasonic device which offers the following benefits: easy implementation in clinical diagnostic routine, fast calculation and an integrated system for image acquisition and measurement connectable to any conventional US-machine, which seems to make the aim of establishing 3D-US monitoring of atherosclerotic disease in daily routine viable.

\section{Materials and methods}

This section describes the key components of the Curefab 3D ultrasound system, consisting of a high-performance computing device, an electromagnetic (EM) tracking system and the ultrasound machine. Next, we provide a brief description of the calibration procedure, before explaining the reconstruction of 3D volumes from a set of tracked US image frames, and present our methods for the evaluation of the system as a whole. These metrics serve as the basis for the results stated in the following sections.

\subsection{D Ultrasound system setup}

Referring to the division into different approaches, the Curefab CS ${ }^{\circledR}$ is described as a freehand 3D ultrasound system with added EM positional sensor information. The system is attached to an ultrasound device for acquiring US image information, while the tracking systems provides pose information for the spatial correlation between the ultrasound image frames. A schematic system layout is depicted in Figure 1.

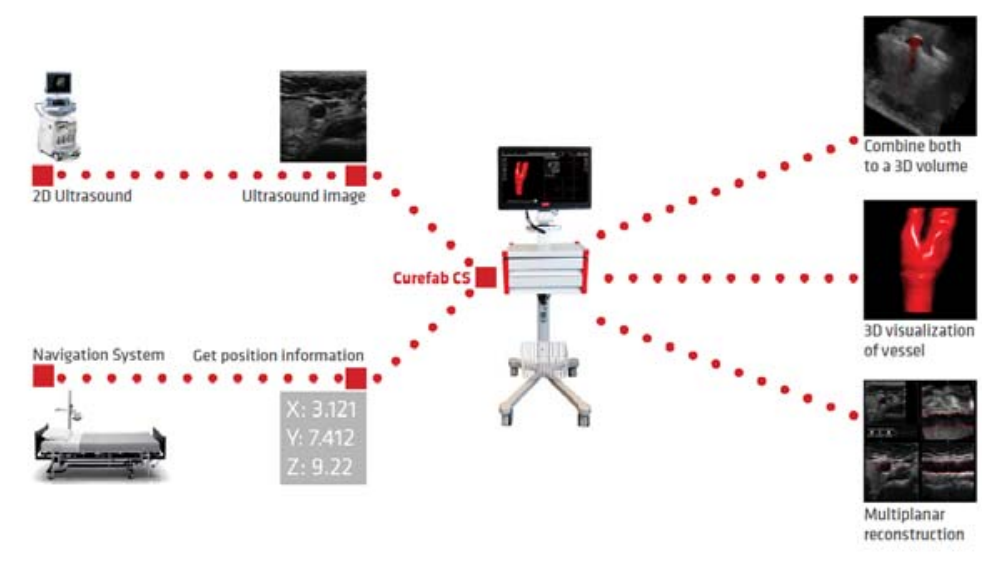

Figure 1. Curefab system overview. Tracking sensors and Ultrasound device are connected to the Curefab system, which enables recording and reconstruction of 3D volumes. 
A high precision frame grabber is used to record the ultrasound image frames, and can be connected to any conventional ultrasound device which has an external analog (VGA) or digital (DVI, HDMI, Display Port) video output.

In order to construct a 3D volume from the set of acquired ultrasound image frames, the position and orientation in 3D space of each and every ultrasound image frame space has to be recorded. To accomplish this sensors are attached to the ultrasound transducer, enabling the tracking of the probe position. For freehand 3D ultrasound systems, optical and EM tracking systems are used most frequently. Although optical tracking provides a higher accuracy in absolute terms, EM tracking is more suitable for ultrasound applications for a couple of reasons. First, optical tracking can become quite awkward quickly since there must always be a clear line of sight between the markers on the ultrasound probe -- held in the sonographer's hand - and the cameras used to track them. EM tracking does not suffer from this problem since the electromagnetic field used to track the sensors passes easily through the sonographer's hand. As a result the EM tracking sensors can be mounted directly ont the ultrasound probe, which is much more convenient for the clinician compared to optical tracking, where at least three markers with as much distance as possible between them need to be mounted in such a way that they hover firmly above the sonographer's hand. The Curefab system uses the 3D Guidance system "drive BAY" (Ascension Technology Corp., Burlington, USA), which according to the specification sheet provides positional accuracy of $1.4 \mathrm{~mm}$ root mean square (RMS) and rotational accuracy of $0.5^{\circ} \mathrm{RMS}$. Two EM tracking sensors are mounted on ultrasound probe to help compensate for the noise inherent in the tracking signal.

Next we discuss the calibration procedure in detail, which aims at finding the spatial transformation between the tracked EM sensor and the ultrasound image frame.

\subsection{System calibration}

The system calibration consists of:

1) Calibrating the ultrasound probe;

2) Synchronizing the incoming $2 \mathrm{D}$ ultrasound images and the tracking data.

In order to calibrate the ultrasound probe, we need to determine the transformation from the sensor mounted on the probe to the $2 \mathrm{D}$ image generated by the probe and displayed on the monitor of the ultrasound machine. The image origin and the sensor can be considered as fixed points on a rigid body (the probe) thus the transformation between them is constant. In order to determine this transformation we scan a specially designed phantom which also has a sensor firmly attached to it. The tracking system delivers the transformation between the phantom and the probe sensor, while the image generated by scanning the phantom is enough to determine the transformation from the phantom to the image origin (see Figure 2).

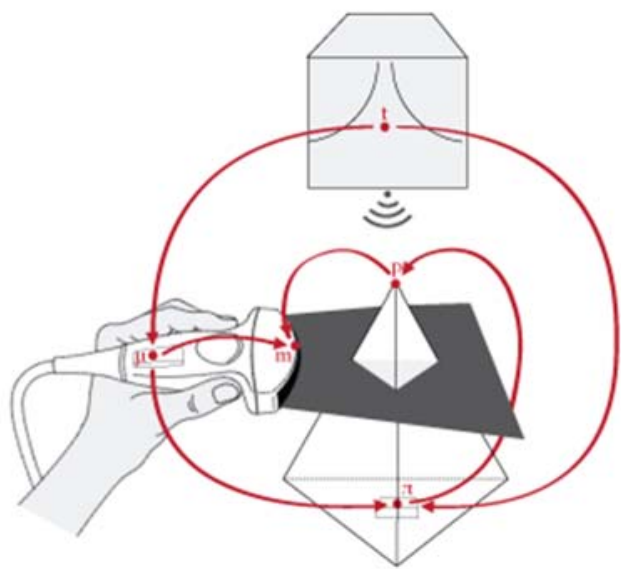

Figure 2. Diagram representing the transformations (arrows) between all coordinate frames considered during the calibration. The world coordinate frame is determined by the electromagnetic field generator-or transmitterdenoted by $t$. Each sensor has its own coordinate frame. The sensor mounted on the probe is denoted by $\mu$, the sensor mounted on the pyramid phantom is denoted by $\pi$. The origin of the pyramid phantom - its tip - is denoted by $\mathrm{p}$, while the origin of the ultrasound image plane is denoted by $m$.

Although a single tracked image of the phantom is sufficient to calibrate the probe, we perform a series of 6 scans, each approximately 10 seconds long, in order to compensate for errors introduced by noise in the tracking data, inaccuracies in 
the automatic segmentation of the phantom images, and the imprecise phantom images introduced by the ultrasound beam width. The series of 6 scans are taken at different locations and orientations relative to the phantom in a symmetric manner so that systematic errors in relative position and orientation will cancel out.

\subsubsection{Description of the pyramid phantom}

The phantom is a trirectangular tetrahedron frame, precision machined from aluminum and covered by a layer of nylon. The edges of the pyramid emanating from the tip are mutually orthogonal and form the basis of a Cartesian coordinate system whose origin is at the tip. A sensor is mounted on the phantom and the transformation from the sensor to the origin of the pyramid coordinate system is determined by the same procedure used to calibrate the probe. When scanned with an ultrasound probe, the nylon skin of the phantom appears as a triangle in the ultrasound image (see Figure 3).
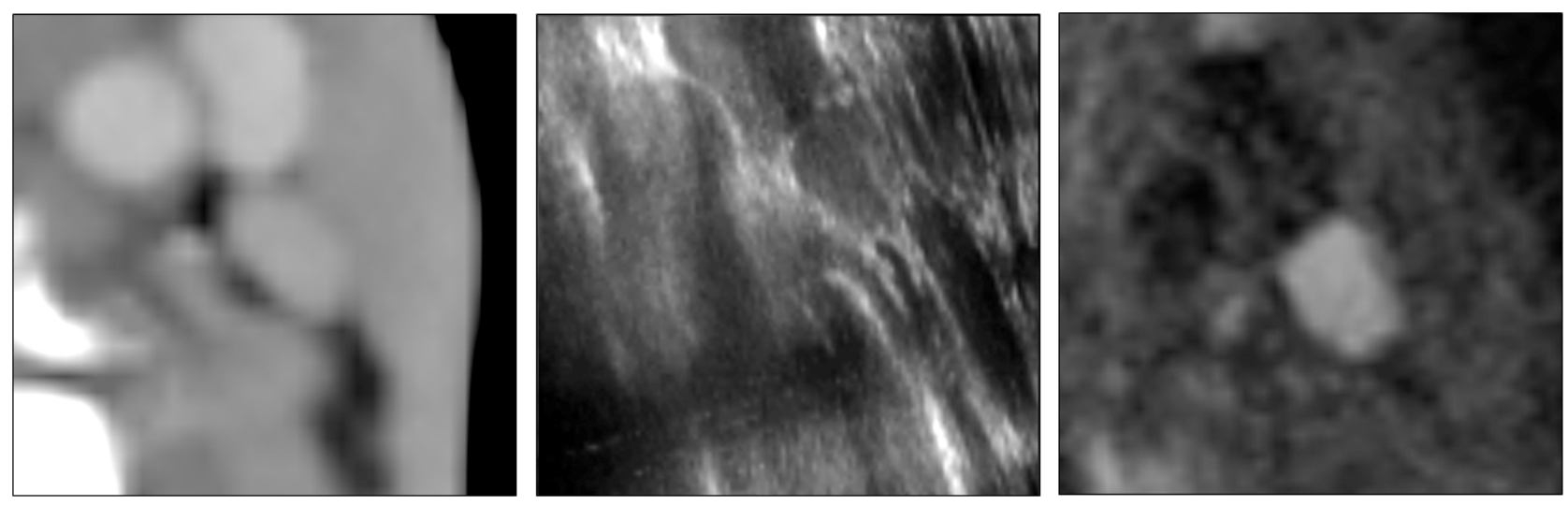

Figure 3. 3D-Ultrasound data (center) manually co-registered with CT data (left) und MRI data (right). Notice the higher resolution of Ultrasound and good visual alignment of circular structures

This triangle is then automatically segmented and from this segmentation it is possible to calculate the transformation from the pyramid origin to the image origin.

The phantom is scanned from each of its 3 axes (the edges emanating from the tip) for both possible orientations of the ultrasound probe. The greatest variation in the probe calibration comes from the alternate orientations of the probe, due to nonzero beam width. The calibration data is filtered to exclude outliers caused by inaccuracies introduced by the data acquisition process.

\subsubsection{Time calibration}

With most ultrasound machines there is a time offset (presumably constant) between the pose data delivered by the tracking system and the image data delivered by the US device via the frame grabber. This is usually on the order of 0 - 200 ms. During the calibration process the phantom and the probe are held as still as possible to minimize these effects. Once the calibration has been performed, we take a U-shaped scan of a tube-shaped phantom and manually adjust the time offset until both halves of the tube phantom are aligned.

\subsection{I mage reconstruction and measurement}

After the calibration of the entire system is performed, reliable pose information can be recorded together with each frame acquired from the ultrasound device. For further calculations the system not only constructs the 3D volume, but also stores all recorded 2D frames with acquired pose information on its storage device. 
For a 3D reconstruction of freehand ultrasound data, several methods have been recently presented ${ }^{[24]}$, where the color values of all voxels within the target volume are estimated by forward or backward interpolation. In terms of forward interpolation, each ultrasound frame is inserted into the 3D volume sequentially, but this involves the risk of "unfilled" holes within the 3D volume, since not all of the pixels from the 2D frame correspond perfectly to 3D voxels in the target volume. In contrast to forward interpolation, backward interpolation uses the color information of the pixel with smallest distance (or a weighted sum of multiple pixels in the neighborhood) to estimate a color value for each target voxel in the 3D volume. Consequently, after the interpolation process no holes are present in the volume. For a detailed description of forward and backward interpolation, the reader is referred to ${ }^{[25]}$.

The Curefab system employs a pairwise backward interpolation between subsequent 2D frames to reconstruct 3D volumes. The method itself is comparable to the one proposed by Wein et al. ${ }^{[26]}$, but instead of selecting all frames with distances smaller than a given threshold to the target voxel, a pairwise interpolation of the volume lying between two images enables a fast reconstruction via parallel processing of the frames on the GPU. Furthermore, by using subsequential frames in time, the reconstruction can be started in parallel to the recording of the images, which provides faster overall recording and reconstruction times.

The algorithm takes two consecutive images with their pose information and estimates the voxels of the target volume which lie between the positions of the frames. For all resulting voxels, the corresponding color values are calculated as a weighted combination of the current color value of the voxel, and the color value belonging to the nearest frame pixel of the two frames. Regarding the distances between target voxel and input frames, a Gaussian window is used to increase the weight of nearer pixels compared to ones with greater distances. This weighting approach was found to provide good and accurate reconstruction results ${ }^{[24,26]}$, while the parallel processing provides fast overall reconstruction.

\section{Results}

To enable a complete evaluation of the whole system, we wanted to address three different key points: the reproducibility of the calibration procedure, the absolute accuracy of the whole system and a direct comparison to CT and MRI imaging modalities. By evaluating all points, an adequate accuracy and practical usability can be verified, while the precision of the calibration is an indicator to the overall quality of the proposed calibration procedure.

For all results except comparison to MRI and CT, a Siemens Acuson S2000 ultrasound system with a 9L4 linear probe at a depth setting of $4 \mathrm{~cm}$ was used and connected to the Curefab CS 3D-ultrasound system. The probe frequency was fixed to $9 \mathrm{MHz}$, focus and other ultrasound image parameters were manually optimized to retrieve good overall image appearance.

\subsection{Calibration precision}

In order to validate our calibration method, we performed a set of 6 calibrations, each consisting of 6 records of our pyramid phantom taken from different positions and angles. In order to be able to compare our method to the performance of other groups we determined the precision of the calibration procedure following the "calibration reproducibility" means used by Hsu et.al. ${ }^{[27]}$. The calibration reproducibility measure is defined as

$$
\mu_{\text {Calib }}=\frac{1}{N} \sum_{i=1}^{N}\left|\sigma T^{m} q-\bar{q}\right|
$$

where ${ }_{\sigma} T^{m}$ is the transformation from the ultrasound image plane coordinate frame $\mathrm{m}$ to the probe sensor coordinate frame $\sigma$ for the $i^{\text {th }}$ calibration, $q$ is the source point of comparison in the image space (either the center or one of the four corner points of the image plane in our case), $\bar{q}$ is the average point of all reconstructed points in the sensor coordinate frame and $N$ is the number of calibrations.

The results for the calibration precision measurements can be seen in Table 1. 
Table 1. Results of our validation measurements. Listed are from top down the calibration precision for center and corner points, the point accuracy as well as the distance accuracy.

\begin{tabular}{lll}
\hline & Mean & Standard deviation \\
\hline$\mu_{\text {Calib }}$ center & $0.16 \mathrm{~mm}$ & $0.23 \mathrm{~mm}$ \\
$\mu_{\text {Calib }}$ top left & $0.23 \mathrm{~mm}$ & $0.34 \mathrm{~mm}$ \\
$\mu_{\text {Calib }}$ top right & $0.18 \mathrm{~mm}$ & $0.21 \mathrm{~mm}$ \\
$\mu_{\text {Calib }}$ bottom left & $0.24 \mathrm{~mm}$ & $0.23 \mathrm{~mm}$ \\
$\mu_{\text {Calib }}$ bottom right & $0.13 \mathrm{~mm}$ & $0.16 \mathrm{~mm}$ \\
$\mu_{\text {Point }}$ & $1.52 \mathrm{~mm}$ & $0.46 \mathrm{~mm}$ \\
$\mu_{\text {Distance }}$ & $0.33 \mathrm{~mm}$ & $0.20 \mathrm{~mm}$ \\
\hline
\end{tabular}

\subsection{System accuracy}

To evaluate the accuracy of the whole 3D-Ultrasound system, the point reconstruction accuracy and the distance accuracy were evaluated, which are methods to compare points or distances measured in 3D space by the system to the real points and distances.

Regarding the point reconstruction accuracy, most other groups use a stylus to measure the "real" position in 3D space ${ }^{\text {[27] }}$. However, we argue that the use of a stylus is sub-optimal for this point retrieval since tracking in any form produces constant error offsets. As a consequence, we defined the real 3D point $P_{W}$ as the average of all measured 3D points by the Curefab 3D-ultrasound system

$$
P_{W}=\frac{1}{M} \sum_{i=1}^{M} P_{W i}
$$

with $M$ equal to the number of measurements and $P_{W i}$ being the measured point for measurementi. For an appropriate quality of the reference point, it is of crucial importance to use records taken from a variety of different orientations to the point phantom. Consequently, potential errors arising from calibration, tracking and other system components, will cancel out. The overall point reconstruction accuracy is defined similar to Hsu, et al. ${ }^{[27]}$ as the absolute difference between the 3D position of one measurement $P_{W i}$ compared to the real position $P_{W}$

$$
\mu_{\text {Point }}=\left|P_{W}-P_{W i}\right|
$$

For our point phantom we attached a single metal wire $(0.5 \mathrm{~mm}$ diameter $)$ vertically to the bottom of a plastic tub filled with water. The tip of the wire was used as target point and scanned from eight diagonal sides to the wire as well as 4 times from the top. From these measurements, the mean point reconstruction accuracy was $1.52 \mathrm{~mm}$, with standard deviation of $0.46 \mathrm{~mm}$.

For distance measurements, a precisely manufactured plastic tube phantom was scanned and the length between fixed landmarks on the tube was measured. As a constant translational scan only tracks the quality of the tracking systems ${ }^{[27]}$, different scanning paths were used, including tilt and rotation of the probe while scanning the phantom, as well as a combined tilt diagonal to the tube phantom. The distance measure $\mu_{\text {Length }}$ was defined similar to the point accuracy as the absolute difference between the real and the measured length. For ten records, the average length measured was $35.89 \mathrm{~mm}$ with a standard deviation of $0.39 \mathrm{~mm}$ between the measurements. Compared to the real length of $36.0 \mathrm{~mm}$, the average $\mu_{\text {Distance }}$ was $0.33 \mathrm{~mm}$ with standard deviation $0.20 \mathrm{~mm}$.

All quantitative measurement results, including calibration precision as well as point and distance accuracy are again listed in a summarized form in Table 1. 


\subsection{Comparison to CT and MRI}

Besides a quantitative evaluation, 3D-Ultrasound records acquired with the Curefab CS system were also compared to CT and MRI scans of patients receiving radiation therapy (see Figure 3) and also to CT data for follow-up investigation of Endovascular Stent Repair of Abdominal Aneurysm (EVAR, see Figure 4). The 3D-Ultrasound data was manually registered to the CT/MRI data for all images presented in this section. The alignment of both image modalities, especially for the EVAR records, showed promising results for future development of diagnostic tools using all image data.
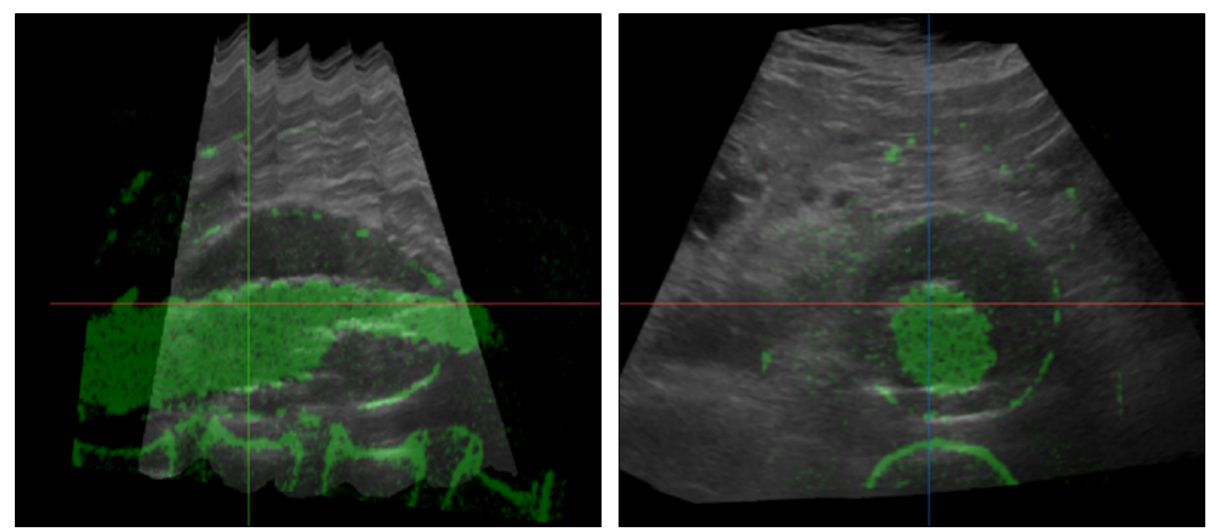

Figure 4. 3DUS and CT manually co-registered and overlaid for an EVAR follow-up investigation. The green semi-transparent shape depicts the CT-data, the rest is 3DUS data

\section{Discussion}

As conventional 2D ultrasound imaging is stretched to its limits when used for monitoring progression or regression of carotid atherosclerotic burden, we focused here on evaluating the reliability of a new freehand and easy to use three-dimensional prospective non-invasive monitoring of carotid atherosclerotic plaques. For this purpose we performed both calibration analysis and validation as well as distance measurements, and clearly demonstrated that distance measurements with Curefab CS are feasible with satisfactory reliability and accuracy.

Our results are in line with findings of previous studies on the accuracy of distance measurements in ultrasonic phantoms which also conclude that three-dimensional ultrasonic distance measurements are sufficiently accurate for clinical application ${ }^{[28-31]}$.

Compared to reviews of recent freehand 3D-Ultrasound calibration methods ${ }^{[21,27]}$, the tested Curefab CS system performs state of the art. The point reconstruction measure retrieved a mean point accuracy of $1.52 \mathrm{~mm}$ in contrast to values ranging from 1.67 to $3.63 \mathrm{~mm}^{[27]}$. Mean total error of distance measurements was $0.9 \%$ with standard deviation $0.56 \%$ in our study, compared with values reaching from about $1 \%$ up to $2.3 \%$ in other studies on this subject ${ }^{[28,30]}$. Given that two-dimensional ultrasound has an error of less than $2 \%$ for distance measurements ${ }^{[28,32,33]}$, we conclude that the distance measurements obtained with Curefab CS are accurate and comparable to those obtained with conventional 2D US devices. Furthermore, precise measurements of distances, which we have proven to be feasible with Curefab CS, are a necessary condition for satisfactory application of planimetrics as the following step, which is currently the standard method for manual measurement of carotid plaque volume. Acquisition of plaque volume for monitoring carotid atherosclerotic burden therefore seems to be an achievable prospective aim with Curefab CS, and there are a multitude of previous studies showing that volume measurements and morphological characterization of carotid plaques are highly promising for non-invasive risk-stratification and genetic research ${ }^{[10,11,16,34]}$ as well as evaluation of a patient's response to therapy ${ }^{[12,13]}$. 
The calibration precision further confirmed the clinical applicability of Curefab CS. Since the calibration method produces highly consistent results with image corner calibration precision values of $0.20 \mathrm{~mm}$ on average (values of 0.47 up to 2.75 $\mathrm{mm}$ for different methods in ${ }^{[27]}$, records can always be acquired on an equal level of accuracy with the system.

Potential shortcomings of the device presented here should not go unmentioned: First of all, it should be pointed out that up to now, real-time acquisition and display of 3D volumes (often referred to as 4D ultrasound) is not possible with Curefab CS. However, 3D-probes which are necessary to that end have to deal with the disadvantage of poor resolution and capture of only small volumes, so that, in our opinion, they cannot be considered as an equal alternative. Second it should be acknowledged that an optical tracking system instead of a magnetically tracked one would have allowed for more accurate calibrations, but the electromagnetic technology used in our study is much more practical for daily use in clinical routine.

\section{Conclusion}

In this evaluation paper, we showed the process workflow from the basic setup of the Curefab CS 3D-Ultrasound system to an accurate 3D reconstruction of the Ultrasound data. The system produces highly accurate image data with a practical and easy setup which delivers a novel level of usability. From the gathered results we draw the conclusion that 3D-imaging with Curefab CS might start off the possibility of accurate visualization, volume measurement, carotid plaque characterization and identification of vulnerable plaques any time soon.

\section{References}

[1] Fenster A, ed. 3-D US Imaging of the Carotid Arteries 2008.

[2] Landry A, Spence JD, Fenster A. Measurement of carotid plaque volume by 3-dimensional ultrasound. Stroke. 2004 ; 35: 864-9. PMid:15017019 http://dx.doi.org/10.1161/01.STR.0000121161.61324.ab

[3] Fenster A, Blake C, Gyacskov I et al. 3D ultrasound analysis of carotid plaque volume and surface morphology. Ultrasonics. 2006; 44 Suppl 1: e153-7. PMid:16844159 http://dx.doi.org/10.1016/j.ultras.2006.06.027

[4] Zwiebel WJ. Duplex sonography of the cerebral arteries: efficacy, limitations, and indications. AJR Am J Roentgenol. 1992; 158: 29-36. PMid:1727355

[5] Sterpetti AV, Schultz RD, Feldhaus RJ et al. Ultrasonographic features of carotid plaque and the risk of subsequent neurologic deficits. Surgery. 1988; 104: 652-60. PMid:3051473

[6] Widder B, Paulat K, Hackspacher J et al. Morphological characterization of carotid artery stenoses by ultrasound duplex scanning. Ultrasound Med Biol. 1990; 16: 349-54. http://dx.doi.org/10.1016/0301-5629(90)90064-J

[7] Fenster A, Lee D, Sherebrin S et al. Three-dimensional ultrasound imaging of the vasculature. Ultrasonics. 1998; 36 : 629-33. http://dx.doi.org/10.1016/S0041-624X(97)00124-8

[8] Fenster A, Downey DB, Cardinal HN. Three-dimensional ultrasound imaging. Phys Med Biol. 2001; 46: R67-99. PMid:11384074 http://dx.doi.org/10.1088/0031-9155/46/5/201

[9] Fenster A, Downey DB. Three-dimensional ultrasound imaging. Annu Rev Biomed Eng. 2000; 2: 457-75. PMid:11701520 http://dx.doi.org/10.1146/annurev.bioeng.2.1.457

[10] Hegele RA, Ban MR, Anderson CM, Spence JD. Infection-susceptibility alleles of mannose-binding lectin are associated with increased carotid plaque area. J Investig Med. 2000; 48: 198-202. PMid:10822900

[11] Spence JD, Ban MR, Hegele RA. Lipoprotein lipase (LPL) gene variation and progression of carotid artery plaque. Stroke. 2003; 34: 1176-80. PMid:12690214 http://dx.doi.org/10.1161/01.STR.0000069160.05292.41

[12] Spence JD, Eliasziw M, DiCicco M et al. Carotid plaque area: a tool for targeting and evaluating vascular preventive therapy. Stroke. 2002; 33: 2916-22. PMid:12468791 http://dx.doi.org/10.1161/01.STR.0000042207.16156.B9

[13] Ainsworth CD, Blake CC, Tamayo A et al. 3D ultrasound measurement of change in carotid plaque volume: a tool for rapid evaluation of new therapies. Stroke. 2005; 36: 1904-9. PMid:16081857 http://dx.doi.org/10.1161/01.STR.0000178543.19433.20

[14] Ludwig M, Zielinski T, Schremmer D, Stumpe KO. Reproducibility of 3-dimensional ultrasound readings of volume of carotid atherosclerotic plaque. Cardiovasc Ultrasound. 2008; 6: 42. PMid:18727816 http://dx.doi.org/10.1186/1476-7120-6-42 
[15] Barnett PA, Spence JD, Manuck SB, Jennings JR. Psychological stress and the progression of carotid artery disease. J Hypertens. 1997; 15: 49-55. PMid:9050970 http://dx.doi.org/10.1097/00004872-199715010-00004

[16] Spence JD, Malinow MR, Barnett PA et al. Plasma homocyst(e)ine concentration, but not MTHFR genotype, is associated with variation in carotid plaque area. Stroke. 1999; 30: 969-73. PMid:10229729 http://dx.doi.org/10.1161/01.STR.30.5.969

[17] Prager RW, Gee AH, Treece GM et al. Sensorless freehand 3-D ultrasound using regression of the echo intensity. Ultrasound Med Biol. 2003; 29: 437-46. http://dx.doi.org/10.1016/S0301-5629(02)00703-2

[18] Gee AH, James Housden R, Hassenpflug P et al. Sensorless freehand 3D ultrasound in real tissue: speckle decorrelation without fully developed speckle. Med Image Anal. 2006; 10: 137-49. PMid:16143560 http://dx.doi.org/10.1016/j.media.2005.08.001

[19] Gee AG PR, Treece GM, Berman L. Engineering a freehand 3D ultrasound system. Pattern Recognition Letters. $2003 ; 24: 757-77$. http://dx.doi.org/10.1016/S0167-8655(02)00180-0

[20] Treece GM, Gee AH, Prager RW et al. High-definition freehand 3-D ultrasound. Ultrasound Med Biol. 2003; 29 : 529-46. http://dx.doi.org/10.1016/S0301-5629(02)00735-4

[21] Mercier L, Lango T, Lindseth F, Collins DL. A review of calibration techniques for freehand 3-D ultrasound systems. Ultrasound Med Biol. 2005; 31: 449-71. http://dx.doi.org/10.1016/j.ultrasmedbio.2004.11.015

[22] Prager RW, Ijaz UZ, Gee AH, Treece GM. Three-dimensional ultrasound imaging. Proc Inst Mech Eng H. $2010 ; 224: 193-223$. http://dx.doi.org/10.1243/09544119JEIM586

[23] Sensen CW HB, ed. Advanced Imaging in Biology and Medicine: Technology, Software Environments, Applications: Springer. 2009.

[24] Rohling R, Gee A, Berman L. A comparison of freehand three-dimensional ultrasound reconstruction techniques. Med Image Anal. 1999; 3: 339-59. http://dx.doi.org/10.1016/S1361-8415(99)80028-0

[25] Jaehne B, ed. Digital Image Processing: Springer. 2005.

[26] Wein W, Pache F, Roper B, Navab N. Backward-warping ultrasound reconstruction for improving diagnostic value and registration. Med Image Comput Comput Assist Interv. 2006; 9: 750-7. PMid:17354840

[27] Hsu P. PR, Gee A., Treece M. . Freehand 3D Ultrasound Calibration: A Review. 2007.

[28] Riccabona M, Nelson TR, Pretorius DH. Three-dimensional ultrasound: accuracy of distance and volume measurements. Ultrasound Obstet Gynecol. 1996; 7: 429-34. PMid:8807760 http://dx.doi.org/10.1046/j.1469-0705.1996.07060429.x

[29] Kurihara TO, T.; Chino, K.; Kanehisa, H.; Fukunaga, T.; Kawakami, Y. Use of Three-Dimensional Ultrasonography for the Analysis of the Fascicle Length of Human Gastrocnemius Muscle During Contractions. International Journal of Sport and Health Science. 2005; 3: 226-34. http://dx.doi.org/10.5432/ijshs.3.226

[30] Zotz RJ, Trabold T, Bock A, Kollmann C. In vitro measurement accuracy of three-dimensional ultrasound. Echocardiography. 2001; 18: 149-56. PMid:11262538 http://dx.doi.org/10.1046/j.1540-8175.2001.00149.x

[31] Tong S, Downey DB, Cardinal HN, Fenster A. A three-dimensional ultrasound prostate imaging system. Ultrasound Med Biol. 1996; 22: 735-46. http://dx.doi.org/10.1016/0301-5629(96)00079-8

[32] Haus AG, ed. The Physics of Medical Imaging - Recording Systems, Measurements and Techniques New York: American Institute of Physics Inc. 1979.

[33] Hagen-Ansert SL, ed. Textbook of Diagnostic Ultrasonics. 1983.

[34] Schminke U, Motsch L, Hilker L, Kessler C. Three-dimensional ultrasound observation of carotid artery plaque ulceration. Stroke. 2000; 31: 1651-5. PMid:10884468 http://dx.doi.org/10.1161/01.STR.31.7.1651 